\section{A novel block chain technology publication model proposal}

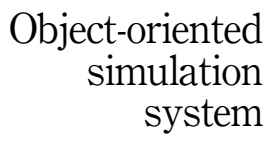

195

Amol Thakre, Fadi Thabtah and Seyed Reza Shahamiri

School of Digital Technologies, Manukau Institute of Technology, Auckland, New Zealand, and

Suhel Hammoud

Department of Information Technology Engineering, Faculty of Engineering, University of Kalamoon, Deirattiah, Syria

\begin{abstract}
Bitcoin is among the highest rated digital crypto-currency in financial investment markets. This technology relies on a backbone of distributed data architecture and peer-to-peer networking model called Blockchain. Unlike the current digital economy, which is governed centrally by financial institution or governments, Blockchain is fully autonomous without any third-party involvement. The exorbitant success of Bitcoin has attracted investors, scholars as well as organizations to peek into this lucrative technology for the possible application to other areas apart from crypto-currency. Blockchain can adopt Smart Contracts, which are digitally enabled contracts that can be executed and enforced fully or partially using pre-defined notions. The aim of this research is to investigate the synergy between Smart Contract and Blockchain to propose a digital framework for an academic paper publication model that has the capability to automate the entire process and challenge the existing system. It can also bring together all the stakeholders under the same system. The proposed model can further hold the stakeholders accountable for breach of contracts and/or reward them for executing the successes of terms pre-configured in the Smart Contract. The proposed model, called Digital Smart Publication or DSP (as referred in the document), is highly secure and ensures balance in distributing rewards to the involved stakeholders while keeping data integrity and security as paramount features.
\end{abstract}

Keywords Bitcoin, Blockchain, Smart Contract, Ethereum, Research publication, Information technology Paper type Original Article

\section{Introduction}

Bitcoin caused a huge excitement in investment market by crossing the un-expected boundaries and reaching 18,000 USD per Bitcoin at the last leg of year 2017. This caused a huge influx among the investors as well as non-investors who demonstrated their willingness

(C) Amol Thakre, Fadi Thabtah, Seyed Reza Shahamiri and Suhel Hammoud. Published in Applied Computing and Informatics. Published by Emerald Publishing Limited. This article is published under the Creative Commons Attribution (CC BY 4.0) license. Anyone may reproduce, distribute, translate and create derivative works of this article (for both commercial and non-commercial purposes), subject to full attribution to the original publication and authors. The full terms of this license may be seen at http:// creativecommons.org/licences/by/4.0/legalcode

Declaration of Competing Interest: The authors declare that they have no known competing financial interests or personal relationships that could have appeared to influence the work reported in this paper.

Publishers note: The publisher wishes to inform readers that the article "A novel block chain technology publication model proposal" was originally published by the previous publisher of Applied Computing and Informatics and the pagination of this article has been subsequently changed. There has been no change to the content of the article. This change was necessary for the journal to transition from the previous publisher to the new one. The publisher sincerely apologises for any inconvenience caused. To access and cite this article, please use Thakre, A., Thabtah, F., Shahamiri, S. R., Hammoud, S. (2019), "A novel block chain technology publication model proposal" Applied Computing and Informatics. Vol. 18 No. 3/4, pp. 195-207. https://10.1016/j.aci.2019.10.003. The original publication date for this paper was $31 / 10 / 2019$.
Received 18 August 2019 Revised 13 October 2019 Accepted 27 October 2019

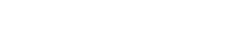


$\mathrm{ACI}$

$18,3 / 4$

196

to invest in crypto-currency. All were quite keen and eager to explore crypto-currency in detail from both financial and technological aspects. The other major reason for Bitcoin popularity was due to the multibillion-dollar transactions globally without any control of central authorities such as financial institutions or government [1]. Meanwhile, cryptocurrency seemed as even more controversial and risky investment from the regulatory issues prospective - it is also surrounded by another concern of taxation due to unclear tax regulations, such as treating it as a commodity or a property, and each country has different views on it but this did not shade down its popularity $[8,9,10,13]$.

Similarly, Smart Contracts is the digitally enabled protocol which can execute a pre-configured term in distributed model. It not only executes and verifies but also enforces the contract terms on all the stakeholders which have participated in the contract; these contracts are irreversible and traceable [6,14]. Smart Contracts is magnetically pulling the attention globally and there is an excitement about how distributed ledgers can support them to achieve the predefined goals. Still in initial stage, Smart Contracts face the challenge of being vulnerable to real world applications as well as lacking definition clarity in the industry [11]. Moreover, there are some legislative constrains too which restrict the use of Smart Contracts in real world, because of its autonomous function the contracts execute automatically without requiring any external authentication. A major concern around Smart Contracts is their implementation as these contracts are highly error prone due to their manual coding [12].

Most academic researchers and other stakeholders involved in the academic publication system are exposed to the stubborn nature of publication industry during some or the other stage of their paper publication $[2,19]$. This traditional publication system has few lacunas which are regulated centrally by editors that cause hindrance in financial gains of the majority of the stakeholders including researchers, reviewers, academic institutions and readers among others. To be exact, in the conventional academic publication system the majority if not all the financial gain of an academic publication is directed to the publisher. Typically, the publisher charges readers, librarians and academic institutions access fees to access academic material including electronic books.

In the last decade a revised publication system based on the open access has emerged [17]. Despite that the open access publication model was created to overcome the primary issues linked with the conventional publication model, such as subscription fee, publishers in the open access publication model still dominate the financial gain as researchers have to pay a certain publication fee to make their academic material accessible free to the readers [3]. In some cases, the fees are in the magnitude of thousands especially when the journal is well established and associated with impact factor. In addition, there are critiques associated with the quality of publication in this publication model [20]. Therefore, the open access publication model has not overcome the primary shortcomings associated with the conventional publication model, i.e. subscription fees, rather it contributed to other serious issues. For example, one of the issues that has emerged from the open access publication model is the biased citation for academic research papers published in journals hosted by unknown publishers that have limited to no quality assurance procedures of handling manuscripts' submission [15]. In fact, there have been recent lawsuits against online publishers such as Sci-Hub that are hosting tens and hundreds of pirated academic journals [18]; unfortunately, the aim of such publishers is attributed to only financial gain rather disseminating quality research. This has caused many research papers with very limited contributions to become highly cited as they have free presence on the internet and can be freely accessed.

Most of the aforementioned issues in the present publication models demand the need for decentralization of the academic publication digital platform, which can enable various stakeholders to contribute towards the publication of academic research papers by 
incentivizing their contributions. This platform should be able to distribute fair tangible and intangible benefits among the stakeholders as agreed in the Smart Contract using Blockchain Technology. The new publication model can be built and integrated within the Ethereum based framework $[4,5,7]$ to address the distribution of the benefits and execute the smart Contract based on the pre-configured conditions. This system will be running on a decentralized mode and the data will be stored in data blocks across the entire network. This will give us a solution to the centrally controlled conventional publication systems and offer shared financial benefits among researchers, reviewers, publishers and editors among others.

Object-oriented simulation system

This paper delivers a proposal in which a novel publication model based on Blockchain technology is introduced that will resolve the issues caused by the conventional publication models mentioned before. Using this model, the stakeholders and participants will be able to leverage the market place and resources in terms of publishing the papers and be incentivized based on their contribution. The incentivization will not stop upon publishing the paper but future benefits will also be distributed among the stakeholders, for example when the paper is citied. This will all be an autonomous process without manual intervention with the help of an Ethereum based framework.

The structure of this paper is as follows: In Section 2, the traditional academic publication model and its shortcomings are described, relevant literature on systems that utilized Blockchain and Smart Contracts are highlighted. In Section 3, the primary issues in the conventional publication models are discussed while Section 4 presents the proposed publication model along with its associated business processes. Lastly, conclusions and future works are given.

\section{The conventional academic publication model}

The publication process of an academic paper is typically operates as depicted in Figure 1. A researcher who wants to publish a paper must submit his research work to the publication house through a journal. Editor is the person authorized from journal to handle the submission process and then to decide over the quality of the paper. Normally, editors initially scan the submitted manuscript for plagiarism, quality, scope and the journal's format. If the research paper fulfills the initial criteria, it then will be forwarded to a list of reviewers. Reviewers will give comments on the paper's contributions, quality and originality. Typically, two to three reviewers are invited to review a research paper where feedbacks and decision are reported. The review process can be blind or double blind and may take multiple rounds until the paper is finally accepted. If the paper is accepted it is then sent to a proof editor, a person with profound knowledge on the technical language in which paper is being published. Proof editors normally perform rigorous checks for alternative words, sentence construction, grammar, citations, references and other related activities. Proof editor at this stage directly converse with the author and instructs them to make the necessary changes. Once all the activities on his front are completed, the research paper falls into next bucket of publication editor who is responsible for publishing the research paper.

Critically analyzing the academic publication model it is clear that the editor of the journal is the key person in the whole process as s/he has the authority to accept or reject the paper. The entire process is centrally approved, and this may sometimes lead to biased decisions especially when reviewers have conflicted opinions when deciding the status of a research paper. The author is sometimes having to give the list of reviewers, but it is usually the editor who decides on the final selected names, this can lead to an area of personal conflict or personal profit. For example, the reviewers suggested by the author may not be necessarily the ones well qualified according to the editor and vice versa. This whole paper's reviewing process seems to revolve around the editor of the journal and his intentions or interest. 


\section{$\mathrm{ACI}$}

$18,3 / 4$

198

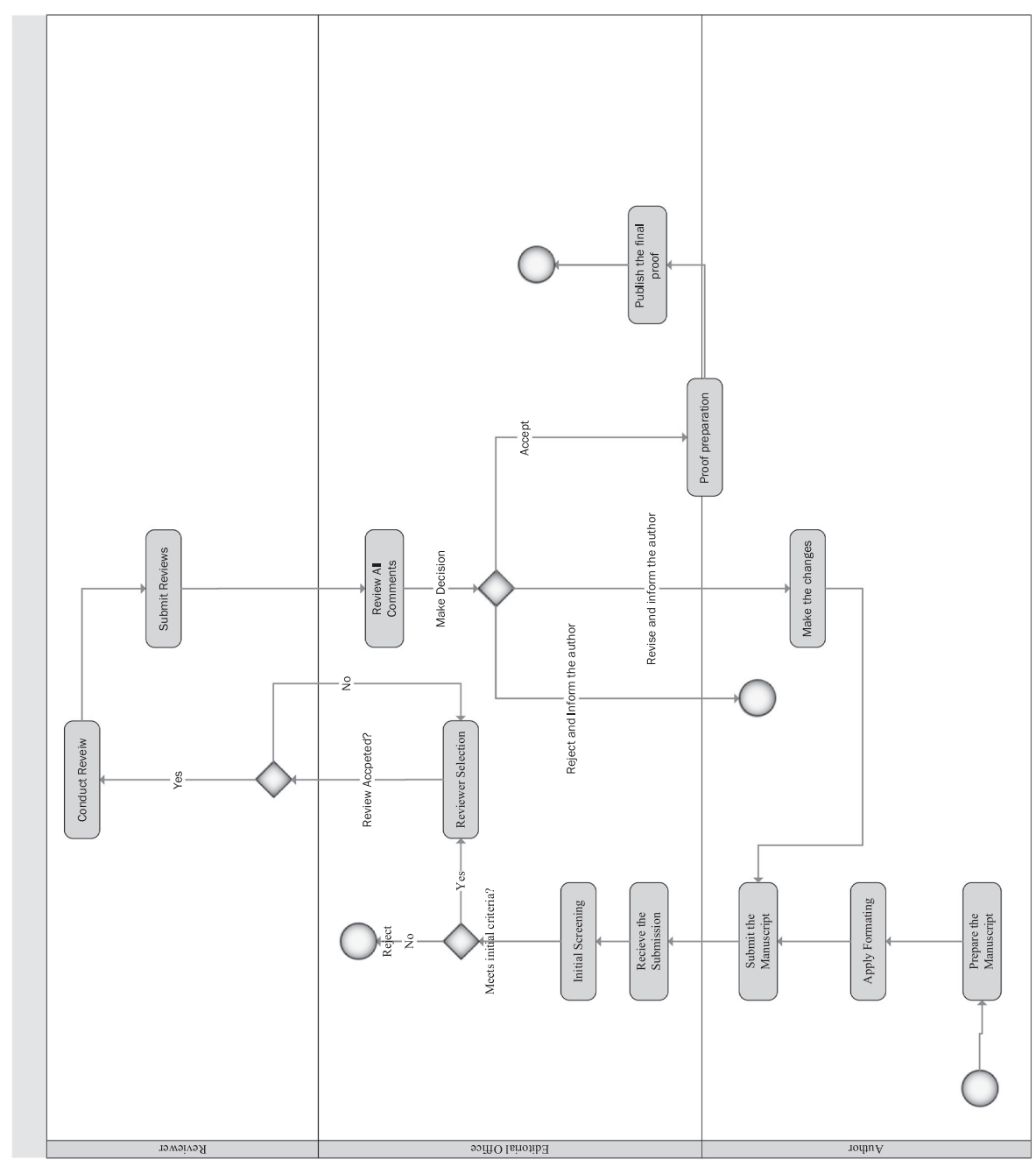

Figure 1.

Academic paper

process in the

conventional

publication model. 
Once the paper accepted and published, the advantage of this publication model is clearly biased towards the publisher, which often charges subscription or publication fees, whereas the key person of this research i.e. the author is only benefitted in an intangible form by the way of recognition. The monetary benefits go directly to the publisher whereas the other key stakeholders including editors, reviewers, readers and authors have no financial gains.

The disadvantageous of the current academic publication model can be summarized as:

- Centralized authority

- Subscription fees (for librarian, institutions, users, etc.) or open access fees (authors)

- Editors of the journal have the primary authority to accept or reject research papers

- Typically, a researcher is considered to be effective only if his work is published in journals with impact factors

- The whole financial benefit goes to the publication house itself and the researchers are not benefitted

- Self-citation issue in a sense that sometimes authors used multiple unnecessary references of their own in a research article

\section{The proposed Digital Smart Publication (DSP) model: overview}

In order to overcome the aforementioned disadvantages of the conventional academic publication model, a fair publication model based on a technology platform which will eradicate the centralized control of the academic research paper publication activities is needed. A platform which will bring all the stakeholders i.e. from researchers to the readers (people who benefit from the research) together to form a network, a digital platform which will enable all to interact with each other directly without any third party involved and each one is incentivized based on the role they play.

The system proposed here will evade the traditional method of publishing and try to enact in a better manner, few of which are mentioned below:

1. A system which will be decentralized or will run on a distributed system, which means the data will be owned by all the stakeholders and without any central authority. To authenticate the data major stakeholders will have to return a true value.

2. No central authority to judge, formulate and decide on the final decisions, a pool of experts can be built up to predict the decision based on their expertise. The system overall will run in an autonomous way and hence no such authority will be involved.

3. The system will be supported by publishers (as one of the stakeholders); publishing on this platform will also gain the market acknowledgement.

4. The system will be running on an incentivizing model: the people will submit their proof of work and hence will be benefitted by doing the work. A token will be distributed against the work which can be claimed anywhere across the similar system or within the system for getting access to the various services.

5. All types of fees collected in various forms will be distributed among all the stakeholders. All the percentages of benefits to the stakeholders will be encrypted in the contracts which will execute automatically without any manual intervention and hence all the process will be transparent and robust.

All these criteria can be achieved by using a new distributed data model and leveraging the Blockchain technology on peer-to-peer network. Integrating this system with Ethereum
Object-oriented simulation system 
$\mathrm{ACI}$

$18,3 / 4$

public network will ensure execution of contracts and distribution of tokens in a robust manner. Many applications are based on Ethereum network and vouch on the framework for execution of Smart Contracts, which will be an essential part of our proposed model.

In particular, the proposed model for publication of academic research, called Digital Smart Publication (DSP), is based on the Blockchain technology with distributed data storage, where all the nodes in the data will carry the information and thus eradicating the use of any central authority while safeguarding the author benefits. In addition, the model avoids the infringement loss of revenue of any party due to implementation of Smart Contracts.

\subsection{Components}

As a Decentralized Autonomous Organization (DAO), DSP's stakeholders will not be bound by formal contracts but by cryptographic tokens and Smart Contracts embedded into the software platform in such as way that there will be no discrepancy of work and related incentives. This is the decentralized and digitalized publication system based on distributed ledger of $\mathrm{P} 2 \mathrm{P}$ systems. In this system, the end user can have direct access to the research paper eliminating the centralized control of the activities and guarding the financial interest of each stakeholder by sharing the profit. The system adopts Smart Contracts to enable the automatic transfer of tokens between the parties automatically based on the pre-conditions defined. This token framework will be based on ERC-20 Ethereum framework that defines the standards for Ethereum tokens. It basically defines the set of rules for Ethereum coins to operate in a larger Ethereum ecosystem which will also enable the p-coins to be compatible with any other system using tokens as well as ERC-20 compliant.

Figure 2 depicts the DSP architecture. DSP components are:

- Data clients: Any person or organization accessing the data on DSP system to access to the research papers. If this research paper is publicly available then the data consumer can access it for free, but if the data owner has no access to it then data consumer will have to pay a fee to have access to this research paper.

- Data Provider: Any person or organization such as publication house contributing towards research publication. The contributions can take different forms such as adding expert's comments or distributing the research work to make it more popular. The name of the person or the organization can be included in the contributors list.

- Data Validator: Any person or organization that validates the entry and acts as a storage client for the distributed database. It can be any of the participants in the network which can validate the Blockchain entry; it can also be the publication house which agrees to share its infrastructure for hosting the research papers and validate the entry when required. In permission-less ledger (Ethereum Network), the incentives will be distributed among validators for doing their job according to the pre-defined conditions. The storage validators will have to prove their stake by avoiding data replication and space time for which the data are available on their infrastructure.

- Token: The tokens are the incentives paid to the stakeholders for the work they do and are automatically processed based on Smart Contracts. DSP system will have its own native tokens called p-token.

- Miner: It is basically a node on the private DSP network which will act as a facilitator for processing the request by other participants in the network and pack it into a Blockchain transaction. The Miner in this case is just a virtual entity and no proof of stake or proof of work is actually done. All the nodes on the network can act as a Miner node.

- DSP Private Network: The private network of DSP stores and obtains the access to data. This network layer will lie upon the Ethereum public network. 


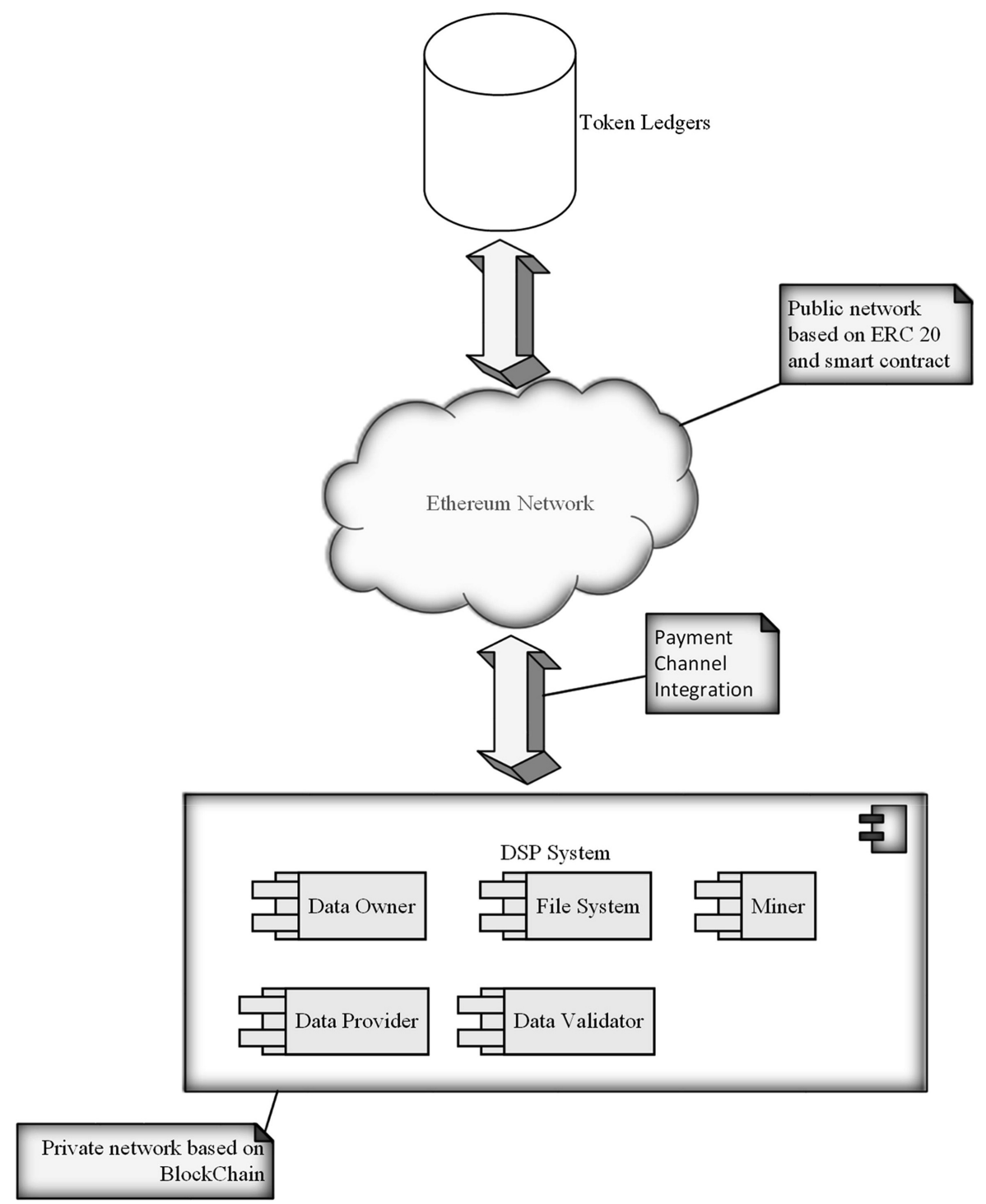

Object-oriented simulation system

201
Figure 2.

The proposed DSP Architecture.

- Ethereum Public Network: An ERC-20 compliant open source system provided by Ethereum to enable token integration, Smart Contracts execution, and maintaining the balances of the token.

As seen in Figure 2, the DSP network will be integrated with the Ethereum network via payment channel integration. Moreover, all the stakeholders will require to join the Blockchain-designed private network. Public access will be provided via a publicly available Ethereum based open source framework that also stores token balances - there will be an 
integration between these private and public networks to enable two-way communications. This integration is vital to establish the contact between the networks when there is some preconfigured state arrives in order to execute the Smart Contract attached to that string for the distribution of p-coins.

\subsection{Finance model}

DSP will initially launch an Initial Coin Offering (ICO) for a certain period - all the registered individuals and entities associated with publication will get certain amount of $\mathrm{p}$-coins as a registration token. The amount of coins offered can differ based on the type of work. Authors or researchers who wish to upload their papers to DSP will have to open an account with the DSP portal initially, and they will be allotted a certain number of tokens as p-coins that will be required to access the DSP system. The value of token will appreciate or depreciate based on the usage of token in the open market according to its popularity, but it will become stale if not used in a predefined time frame that can be embedded in the Smart Contracts. Once the ICO period is over the new user will have to pay the fees for opening an account and he will be allocated the tokens based on the current value of p-coin. The integration with ERC-20 will enable the exchange of these p-coins with other coins such as Bitcoin [16]. Similarly, the p-coins can be exchanged/spent on other ERC-20 compliant systems. This process is further explained in the next section.

\section{The proposed Digital Smart Publication model: process}

If a researcher is willing to publish his paper on DSP system, he will have to initially be part of the DSP network. A standard procedure will be attached to the system enabling the user to create his user credentials. Moreover, one time joining fee will be required to be paid at the time of registration as explained before. Once the user is created in the DSP system, he will be emailed the details of his credentials. A digital wallet will also be created within the system with unique id and the balance of token available and visible to the user once s/he logins. After the user submits the paper to the DSP system, an e-mail will be sent as the token of confirmation against this submission. The system will itself check for plagiarism and other necessary checks at initial level and categorizes the work into a designated category i.e. science, math, business, medical, etc. based on the criteria and options chosen by the researcher during the paper submission stage and natural language processing techniques.

Once the author submits the paper it will be available on editor's dashboard. He will further push the research paper for review. The DSP system automatically looks for the active reviewers based on the research topic and suggests the probable reviewers. The editor can confirm the result suggested by DSP system and submit the research paper for the review. Alternatively, he can change the reviewers and allocate it to others, but he will have to give a reason for it. He can also allocate this research paper to the reviewers through some publication house, providing the publisher and reviewers are already DSP stakeholders so they can be compensated through giving credits apart from distribution of p-coins. Reviewers in this case will be of two types:

1. Participating as a reviewer directly on DSP network - token distributed directly to them, or

2. Participating as a reviewer through publication house - token distributed to the publication house.

Once the reviewers submit their comments and decisions, it will fall into editor's bucket once again with the flag of Stage-I (Review completed), and the reviewers will be rewarded with token according to the criteria marked in the Smart Contract. In case the paper is rejected by the reviewers because of quality concerns, the researcher can be given two options: 
1. Self-publish - wherein the user can still take services offered by the DSP system and other users for self-publication. The user can use such services to improve the paper. In this case they will be able to coordinate with each other and once the work is done, the p-coins will be paid to all contributors deducting it from the researcher's account.

\section{Object-oriented simulation system}

2. Token-Refund - if this option is taken the user has the liberty to take the balance of his token back and convert it into the legal tender either by transferring it to his/her own bank account or s/he can use it to render other services offered by the DSP system or on any other ERC-20 compliant portals. In this case the refund will be minus the reviewers and initial processing fees (if applicable, see below).

The reviewers will be basically predicting the success of the paper, and the success of it will depend upon the number of times the paper has been accessed and cited at later stage of publication. The majority of 'Accept' decision will entitle the paper towards publication. All the reviewers who predict the correct outcome will be rewarded with tokens while those who predict the wrong outcome will have to pay in terms of token. This credit and debit of tokens will be an automatic outcome already pre-configured in the Smart Contract.

The second stage will be proof reading (Stage-II). At this point the editor will publish the paper over the network and ask the registered proofreaders within DSP for proofreading services. The editor agrees to share the research paper with the selected proof editor(s) or select another one. After the proofreader's recommendations are ready, she can contact the author on a common platform for making the changes or alternately make changes to the document and upload it to the DSP file system. All the changes can be tracked as it will be stored and traced in each stage. Once the proof-reading phase is complete, the proof editor submits the work to the DSP file system with final comments. This will also publish the research report again into the editor's dashboard for further action. The status of research paper will be flagged as Stage-II (Proof reading completed).

Flagging of document as Stage-II complete triggers the publisher that enables the system to suggest possible publication editors - similar to the previous stages the editor can go ahead with the system recommendations or select manually. Once the publication editor is attached to the research paper, he can interact with the author directly on a common platform for finalizing the publications. The research paper can be simultaneously published through various digital channels like author's personal website or various publication houses if they have any credit attached to the research paper such as participation during the review process or hosting the files on their servers. Each stakeholder will get benefits in form of tokens for all their activities and all the future referencing to this research paper. Figure 3 summarizes this procedure.

These token values can be distinguished based on the type of work such as usage tokens or work tokens. Tokens are basically like the shares in companies and reflect the ownership in the company but at the same time, it can be used to pay against any services offered on the network. Like the participants engaging themselves, data validators can be given better benefits because they will be sharing their infrastructure for the storage and authenticating the data when required. Once the paper is published, data clients (i.e. registered end users) will be able to access the paper as shown in Figure 4. Free papers do not require any token, but clients need to spend tokens for premium papers.

\section{Discussion and conclusion}

The proposed DSP system can help in changing the market perspective of traditional publication methods. It brings opportunities to not only eradicate the traditional way of academic research paper publication but also incentivize all stakeholders by enabling them to earn for the work they do via token distribution. The researcher will be continuously gaining 


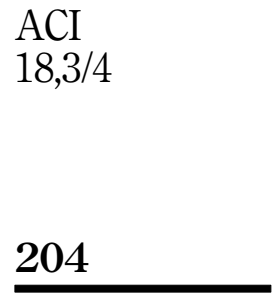

Figure 3.

DSP Stages.

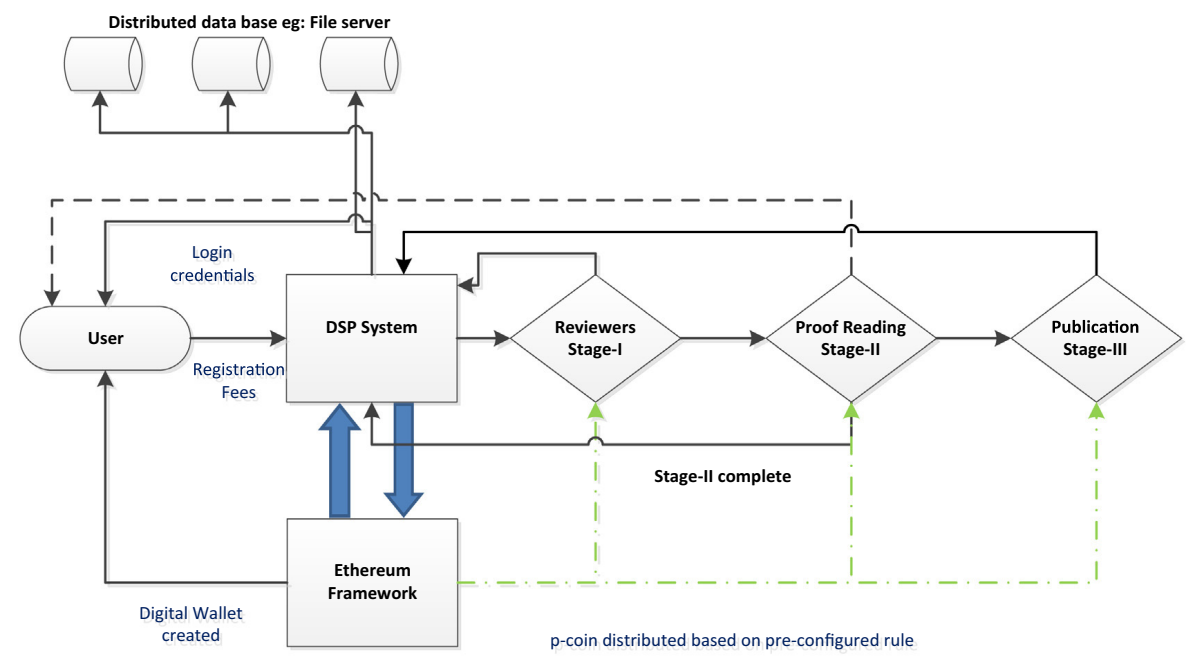

the benefits when the paper is being referred or citied. Moreover, there will be no central authority to make decision and all the payments will be transparent and direct. Using ERC-20 compliant Ethereum framework helps DSP in completing flawless transactions across platforms compatible with Ethereum public network.

Moreover, Smart Contracts based token distribution ensures the tokens are distributed among the stakeholders when certain criteria are met. DSP also ascertains that the benefits are long lasting as transfer of tokens will take place whenever the pre-defined conditions are satisfied. Likewise, Blockchain based data will help to ensure the decentralization of control of the system and also the authentication of data which means there will be no overriding of data once they are committed. Smart Contracts handle the configurations of all the conditions to enable the system to be more autonomous and self-govern.

The benefits gained due to the digitalized presence of DSP can be given back to the publication community in various ways. The educational institutions or the organizations promoting the various learning methods throughout the globe can be given access to the system for recurring minimum pricing in favor of using their infrastructure as a file server. Librarians will have access to the online platform to facilitate manual maintenance of the books - they can also participate in making a paper successful by predicting its success.

To summarize, the key advantageous of DSP are:

- Eradicating the centralized control brings more visibility and transparency over the process and helps the editors to make more democratic decisions.

- Incentivizing based on token distribution ensures benefits for all stakeholders.

- The use of Smart Contracts enables the token distribution based on pre-configured rules.

- Recurring benefits to all stakeholders every time the paper is referred.

- Since the stakeholders' benefits depend on the success of the paper, they all work harder to improve the quality of the paper hence the overall quality of research publications will improve. 


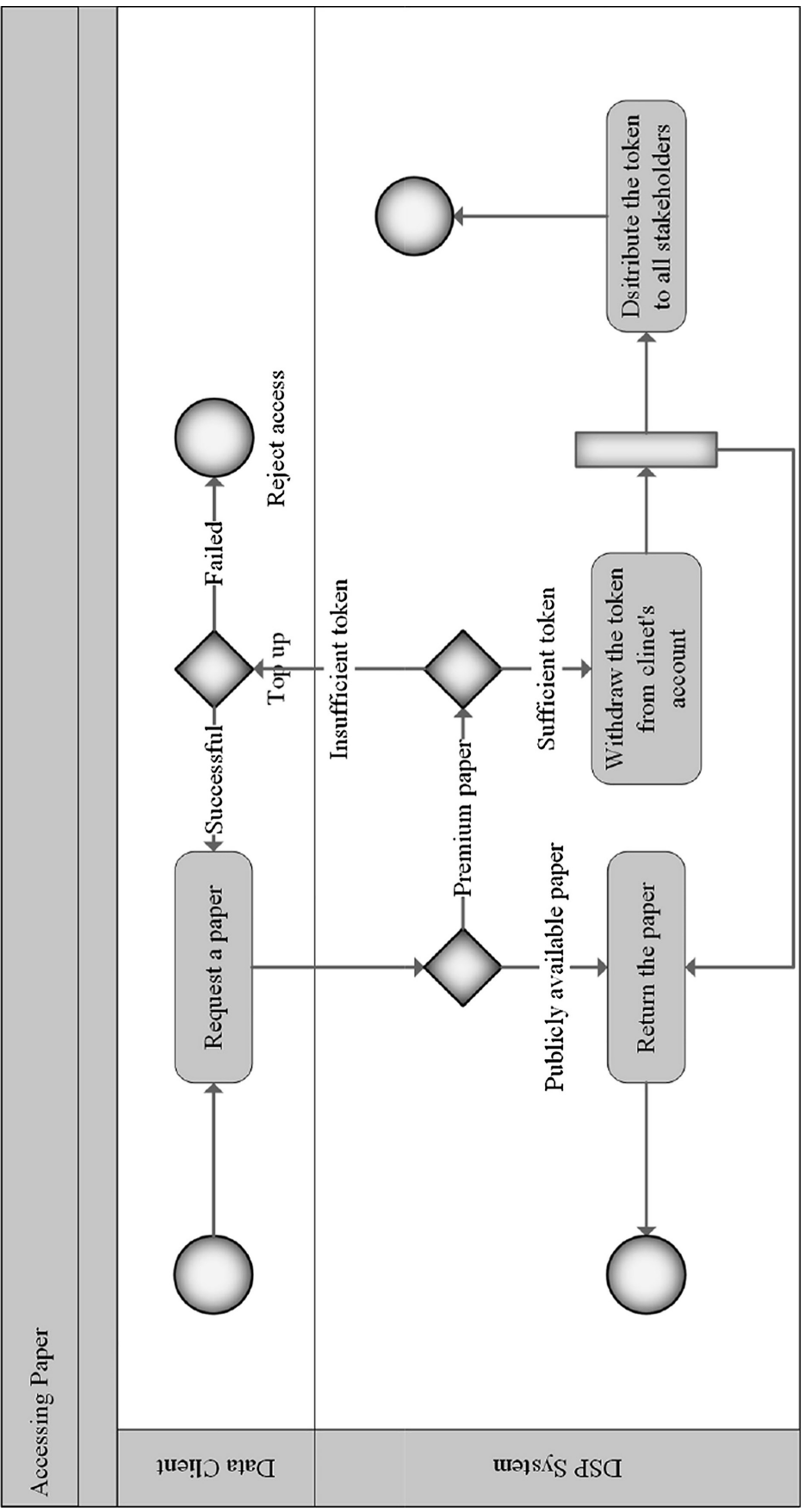

Object-oriented simulation system

205

Figure 4

Research Paper Access by End Users. 
$\mathrm{ACI}$

$18,3 / 4$
- Digital presence of the system can open up the new avenue of crowd funding if more practical research needs to be done in any of the subjects. DSP system will support this.

- Applying an Ethereum framework will allow the p-tokens to be used for gaining the service offerings on other systems.

Overall, DSP can generate lucrative results for all the peers in the network. The use of public network will assure for the future integration with other systems too. However, there are still some unexplored areas in the Blockchain technology due to lack of research in the area which may cause a blockage in successful implementation and adoption of this publication model. Followings are the key areas which we think that any realization of DSP should consider:

1. The research paper privacy should be guarded by this technology as the openness of DSP may make unpublished works open to all participants beforehand while the paper is being processed for publication. Thus, any DSP realizations must guarantee the privacy and confidentiality of the submissions.

2. During publication there are chances of duplicate work being published in the offline model via the traditional systems. While DSP will be guarded by Smart Contracts, it does not hold true outside this virtual ecosystem. This is an issue that existing systems also need to deal with.

3. Smart Contracts are not well accepted in real world. This constrain is still at initial stage and other considerations need to be discussed over this subject. There is no compliance for Smart Contracts within judiciary systems in real world and hence any fraudulent matters cannot be handled in the way it probably should be.

4. It will be difficult for the traditional publishers to adopt DSP and share their financial benefits with other stakeholders. It will be a complex mission to change the mindset of people being settled in the traditional model wherein the whole process is centralized and implementing the solution like this can vary their core interest of being in authority. Nevertheless, this can be compensated by distribution of p-coins which can attract them to this region but it may still remain a risky decision for them.

5. An autonomous and self-propelling system based on distributed ledger model will ensure unbiased decisions, prompt payments and authenticity of work.

\section{References}

[1] Arvind Narayanan, Joseph Bonneau, A.M. Edward Felten, Steven Goldfeder, Bitcoin and cryptocurrency technologies, Network Secur. (2016), http://dx.doi.org/10.1057/palgrave.jit. 2000080.

[2] S.A. Azer, D.M. Dupras, S.A. Azer, Writing for publication in medical education in high impact journals, Eur. Rev. Med. Pharmacol. Sci. (2014).

[3] C.W. Bailey, What is open access?, in: Open Access: Key Strategic, Technical and Economic Aspects, 2006, http://dx.doi.org/10.1016/B978-1-84334-203-8.50002-9.

[4] V. Buterin, in: Ethereum White Paper: A Next Generation Smart Contract \& Decentralized Application Platform, 2013, http://dx.doi.org/10.5663/aps.v1i1.10138.

[5] V. Buterin, Ethereum state transition function, Etherum (2018), http://dx.doi.org/10.5663/aps. v1i1.10138.

[6] B. Cant, A. Khadikar, A. Ruiter, et al., Smart contracts in financial services: getting from hype to reality, Capgemini Consult. (2016), http://dx.doi.org/10.1006/prep.2001.1558. 
[7] M. Crosby, Nachiappan, P. Pattanayak, et al., Blockchain technology beyond bitcoin, Blockchain Object-oriented Technol. (2015), http://dx.doi.org/10.1515/9783110488951.

[8] P.D. Devries, An analysis of cryptocurrency, bitcoin, and the future, Int. J. Bus. Manage. Commerce (2016).

[9] P. Härle, A. Havas, A. Kremer, et al., The Future of Bank Risk Management, 2015, McKinsey Working Papers on Risk.

[10] S. Huckle, M. White, Socialism and the blockchain, Future Internet (2016), http://dx.doi.org/10. 3390/fi8040049.

[11] M. Kõlvart, M. Poola, A. Rull, Smart contracts, in: The Future of Law and ETechnologies, 2016, http://dx.doi.org/10.1007/978-3-319-26896-5_7.

[12] L. Luu, D.-H. Chu, H. Olickel, et al., Making smart contracts smarter, in: Proceedings of the 2016 ACM SIGSAC Conference on Computer and Communications Security - CCS'16, 2016, 2016, http://dx.doi.org/10.1145/2976749.2978309.

[13] J. Mattila, The challenges of banking regulation and supervision, J. Econ. (2014).

[14] E. Mik, Smart contracts: terminology, technical limitations and real world complexity, Law Innov. Technol. (2017), http://dx.doi.org/10.1080/17579961.2017.1378468.

[15] M. Norris, C. Oppenheim, F. Rowland, The citation advantage of open-access articles, J. Am. Soc. Inform. Sci. Technol. (2008), http://dx.doi.org/10.1002/asi.20898.

[16] N. Satoshi, S. Nakamoto, Bitcoin: a peer-to-peer electronic cash system, Bitcoin (2008), http://dx. doi.org/10.1007/s10838-008-9062-0.

[17] J.P. Tennant, F. Waldner, D.C. Jacques, et al., The academic, economic and societal impacts of Open Access: an evidence-based review, in: F1000Research, 2016, http://dx.doi.org/10.12688/ f1000research.8460.3.

[18] J.P. Tennant, J.M. Dugan, D. Graziotin, et al., A multi-disciplinary perspective on emergent and future innovations in peer review, in: F1000Research, 2017, http://dx.doi.org/10.12688/ f1000research.12037.3.

[19] B. Warren, Current challenges and choices in scientific publication, Proceedings (Baylor University. Medical Center) 16(4). Baylor University Medical Center: 401-4, 2003 (accessed 11 February 2019) http://www.ncbi.nlm.nih.gov/pubmed/16278757.

[20] M.G. Weinberger, C.S. Gulas, M.G. Weinberger, et al., How to critique a journal article, Int. J. Advertising (2013), http://dx.doi.org/10.1017/CBO9781107415324.004.

\section{Corresponding author}

Fadi Thabtah can be contacted at: Fadi.fayez@manukau.ac.nz

For instructions on how to order reprints of this article, please visit our website:

www.emeraldgrouppublishing.com/licensing/reprints.htm

Or contact us for further details: permissions@emeraldinsight.com 